\title{
Monitoring of underground coal fires using thermal infrared data
}

\begin{abstract}
The potential utility of thermal infrared and short wavelength infrared data for detecting and mapping sub-surface high temperature sources is analysed. In this study, NOAA-9 AVHRR data and Landsat-5 TM data were used to detect and map sub-surface coal fires. Brightness temperature depicted by AVHRR band 3 illustrated high thermal anomalies in the suspected area. Due to the relatively low spatial resolution of the AVHRR data, only TM data is used in detailed analysis. The short wavelength infrared sensors (bands 5 and 7) have been used to locate the positions of the most intense burning. The thermal band (band 6) has been useful in distinguishing gross thermal anomalies from the background of solar warming, The resultant surface temperature anomalies are compared to surface temperatures derived from thermal infrared aerial survey and ground measurements. Correlation of these data indicate that the relatively coarse resolution of the thermal TM data enabled the detection, delineation and quantifying of sub-surface coal fire zones. However, the capability of the short wavelength infrared bands to locate the position of the fire fronts is only preliminary. The research shows that the information gathered from the TM data could only be used as a basis for planning the detailed ground geothermal operation. The investigation also reveals the potential capability of the AVHRR band 3 to detect sub-surface high temperature sources such as coal fires.
\end{abstract}

